\title{
Laddering em pesquisa de marketing
}

\section{Laddering in marketing research}

Tânia Modesto Veludo-de-Oliveira ${ }^{l}$

Ana Akemi lkeda

Resumo

O artigo discute a técnica de pesquisa laddering em marketing, considerando a proposta de renovação de conhecimentos sobre metodologia na área. Para isso investiga diversos estudos, artigos e pesquisas que tratam do assunto em nível nacional e internacional. 0 conceito de cadeias meios-fins é abordado pela estrutura teórica que fundamenta a técnica, ligando atributos, conseqüências percebidas do consumo e valores pessoais relativos a um produto. 0 artigo segue com a explicação dos procedimentos de campo, como a entrevista e a análise dos dados. A laddering é um instrumento de pesquisa qualitativa bastante útil e poderoso, mas ainda pouco utilizado tanto por acadêmicos como por profissionais de mercado, no Brasil, provavelmente por desconhecimento e pouca divulgação. Cabe, portanto, uma maior disseminação de seu uso.

Palavras-chave: laddering; pesquisa de marketing; cadeia meios-fins

\section{Abstract}

The objective of this paper is to discuss laddering technique in marketing, considering the proposal of renewing knowledge on research methodology. It investigates national and international studies, articles, and researches that approach the subject. The means-end theory that grounds the technique, linking attributes, consequences and values related to a product, is presented in the article. Common practices in the field research are presented as well, emphasizing data collection and analysis. Although very powerful, laddering is still underutilized for both academics and practitioners, probably for lacking of knowledge and dissemination. It is important to propagate the use of this technique in academic and market researches.

Keys words: laddering, marketing research; means-end chain

\section{Introdução}

A laddering é uma técnica apropriada para estudos de marketing e áreas relacionadas, sendo que seu uso mais comum tem sido aplicado em comportamento do consumidor. Nesse domínio, a laddering vem sendo bastante aplicada para explorar atributos do produto, conseqüências percebidas do consumo e estados-fim percebidos (LANGERAK; PEELEN; NIJSSEN, 1999). O objetivo deste artigo é apresentar a metodologia de laddering como um instrumento de pesquisa em marketing. Pretende-se cobrir os principais conceitos, explicar como são feitos a entrevista e os procedimentos de análise, além de discutir os problemas encontrados no uso dessa técnica.

\footnotetext{
${ }^{1}$ Doutoranda da Cardiff Business School (UK) e bolsista da CAPES. Mestre em Administração pela Faculdade de Economia, Administração e Contabilidade da Universidade de São Paulo (FEA/USP). Endereço: Rua Dr. Vasco de Andrade, 94 apto. 201 - Uberaba - Minas Gerais -- CEP: $38017-200$ - Brasil tveludo@usp.br

2 Professora Associada da FEA/USP e vice-coordenadora do MBA Marketing da FIA. Endereço: Rua Luciano Gualberto, 908. Sala E-104. 05508-900. Cidade Universitária - São Paulo (SP)- Brasil. anaikeda@usp.br

Artigo submetido em fevereiro de 2006 e aceito em fevereiro de 2007
} 


\section{Laddering em pesquisa de marketing}

A laddering foi originalmente criada no campo da psicologia, com Kelly (1955) e Hinkle (1965), migrando para a área de marketing, anos depois de seu surgimento. Hinkle desenvolveu seus estudos de doutorado sob a supervisão de Kelly, na Ohio State University, aplicando a "teoria do construto pessoal", idealizada por seu orientador para acessar construtos de alto nível de abstração. Para tanto, fez-se necessário o desenvolvimento de uma ferramenta de pesquisa capaz de revelar os sistemas de significados pessoais dos indivíduos, que foi chamada laddering (BANNISTER; MAIR, 1968). Do termo "ladder", que em inglês significa "escada", depreende-se o principal sentido da técnica: passar do nível mais superficial ao mais profundo, do mais concreto ao mais abstrato, do mais tangível ao mais conceitual, por meio de questões abertas, e assim descobrir insights e chegar à raiz do problema.

No início da década de 80, Gutman (1982) se interessou pela técnica e vislumbrou possibilidades de sua aplicação na área de marketing. Desenvolveu um arcabouço teórico para fundamentá-la no contexto mercadológico, conhecido por "teoria meios-fins" e disseminado principalmente por meio de seu trabalho em parceria com Reynolds (REYNOLDS; GUTMAN, 1988). Assel (1995) argumenta que Gutman (1982) tornou as teorias de valores pessoais mais aplicáveis ao marketing ao adicionar o elemento "atributos do produto" como uma forma de alcançar as metas de consumo. Em marketing, portanto, a laddering assenta-se no modelo de cadeias meios-fins, objeto do tópico seguinte. O Quadro 1 mostra diversos estudos que discutem a laddering ou que conduziram pesquisas por meio dela em marketing, o que inclui investigações sobre serviços, bens físicos, idéias, pessoas e lugares. 
Quadro 1. A laddering em estudos de marketing

\begin{tabular}{|c|c|}
\hline Estudos & Pontos discutidos \\
\hline Reynolds e Gutman (1988) & Explicação da laddering e de suas etapas. \\
\hline Lastovicka (1995) & Utilização do software LADDERMAP. \\
\hline \multirow[t]{2}{*}{ Grunert e Grunert (1995) } & Validade da laddering. \\
\hline & Aprimoramento da laddering por meio de: \\
\hline Valette-Florence e Rapachi (1991) & - análise de correspondência. \\
\hline \multirow{2}{*}{ Lin $(2002)$} & - análise fatorial e regressão linear. \\
\hline & Uso da laddering para criação de estratégias de: \\
\hline Reynolds e Whitlark (1995) & - comunicação. \\
\hline Gengler e Reynolds (1995) & - comunicação. \\
\hline Botschen, Thelen e Pieters (1999) & - segmentação de mercado por benefícios. \\
\hline \multirow[t]{2}{*}{ Vriens e Hofstede (2000) } & - segmentação de mercado. \\
\hline & Comparação da laddering com outras técnicas, como: \\
\hline Woodruff e Gardial (1996) & - grand tours. \\
\hline Wansink (2000) & - prototyping. \\
\hline \multirow[t]{2}{*}{ Christensen e Olson (2002) } & - zaltman metaphor-elicitation technique (ZMET). \\
\hline & Pesquisas conduzidas a partir da laddering, enfocando: \\
\hline Bagozzi e Dabholkar (1994) & - reciclagem. \\
\hline Gengler, Howard e Zolner (1995) & - pessoal de vendas em revendedoras de automóveis. \\
\hline Gutman (1997) & - bebidas. \\
\hline Gengler, Mulvey e Oglethorpe (1999) & - amamentação de bebês. \\
\hline Botschen, Thelen e Pieters (1999) & - pessoal de vendas em lojas de roupas. \\
\hline Goldenberg et al. (2000) & - cursos de 'rapel'. \\
\hline Bagozzi e Dabholkar (2000) & - presidente Bill Clinton. \\
\hline Kennedy, Goebel e Deeter-Schmelz (2000) & - veículos (SUV). \\
\hline Vriens e Hofstede (2000) & - iogurtes. \\
\hline Thyne (2000) & - museu. \\
\hline Wansink $(2000,2003)$ & - sorvete Häagen-Dazs. \\
\hline Dibley e Baker (2001) & - salgadinhos. \\
\hline Allen $(2001)$ & - carro Toyota Corolla. \\
\hline Coolen e Hoekstra (2001) & - casas/ moradia. \\
\hline Leão e Mello $(2001,2002)$ & - jornal virtual. \\
\hline Lin $(2002)$ & - lojas de conveniência. \\
\hline Manyiwa e Crawford (2002) & - café da manhã. \\
\hline Ignácio (2003) & - alimentos. \\
\hline Orsingher e Marzocchi (2003) & - hotel. \\
\hline Veludo-de-Oliveira (2003) & - serviços educacionais. \\
\hline
\end{tabular}

Fonte: representação proposta pelas autoras.

\section{$A(s)$ cadeia(s) meios-fins}

Ainda que possa ser aplicada a outros aspectos comportamentais estudados em marketing, como satisfação, a teoria meios-fins está ligada principalmente a pesquisas sobre o valor para o cliente, o que, segundo Woodruff e Gardial (1996), está profundamente relacionado ao uso de técnicas qualitativas - dessa forma a laddering apresenta-se como uma boa alternativa.

Essa teoria estabelece uma associação entre as características do que está sendo usado, consumido ou comprado, ou seja, os atributos do produto (A), com os motivos que estão por trás disso, isto é, as 
conseqüências comportamentais $(\mathrm{C})$ e os valores pessoais $(\mathrm{V})$. Os atributos são os aspectos mais tangíveis do produto, enquanto que as conseqüências referem-se aos benefícios ou aos custos de usá-lo, consumi-lo ou comprá-lo (GENGLER; MULVEY; OGLETHORPE, 1999) e os valores, finalmente, são os estados finais da existência buscados pelo ser humano, como paz e contentamento.

De acordo com o modelo, os "meios" são os produtos ou as atividades nas quais as pessoas estão envolvidas (A ou C), e os "fins" são os estados valorados da existência (V). Traça-se, então, um caminho para formar o encadeamento A-C-V, onde "meios" levam a "fins", ou seja, atributos de produtos (A) levam a conseqüências (C) que, por sua vez, ajudam a alcançar os valores pessoais do indivíduo (V). São quatro as suas suposições principais: (a) os valores são fatores dominantes no padrão de compras dos consumidores; (b) os consumidores agrupam produtos baseados na função de satisfazer valores; (c) todas as ações têm conseqüências e (d) há uma ligação entre conseqüências e atributos dos produtos (GUTMAN, 1982).

Esse modelo fornece um caminho relativamente simples para a caracterização das inter-relações cognitivas entre as dimensões: atributos, conseqüências e valores pessoais. As características dos produtos ganham relevância ou significado para os consumidores devido à associação que estes fazem com as conseqüências advindas de seu uso e, conseqüentemente, com o alcance de seus valores pessoais.

Olson e Reynolds (1983) propuseram a subdivisão dessas três dimensões de forma que os atributos possam ser avaliados como concretos e abstratos, as conseqüências como funcionais ou psicológicas e os valores, como instrumentais e terminais, chegando-se a seis níveis de abstração que se relacionam hierarquicamente. Os atributos concretos são geralmente identificados pelas características físicas do produto, como peso (VRIENS, HOFSTEDE, 2000), tamanho e gosto (WANSINK, 2000), e os abstratos são características mais subjetivas, como qualidade (BOTSCHEN; THELEN; PIETERS, 1999, LEÃO; MELLO, 2001) e beleza. As conseqüências funcionais são provocadas pelo produto consumido (VALETTE-FLORENCE; RAPACCHI, 1991) e são os resultados da experiência direta do consumo, como saciar a sede. As conseqüências psicológicas são os resultados emocionais ou sociais do consumo do produto, como imaginar o que os amigos pensariam ao ver o indivíduo consumindo algo. Os valores instrumentais, segundo Rokeach (1973), são os modos para se chegar aos valores terminais, que são os estados finais da existência - o afeto e a ternura, vistos como valores instrumentais, poderiam ajudar a conquistar os valores terminais de felicidade e amor, por exemplo. A Figura 1 mostra exemplos dos seis níveis de abstração da cadeia ampliada para efeitos de ilustração, uma vez que nem sempre poderão ser identificados todos os níveis. 
Figura 1. Níveis de abstração da cadeia meios-fins ampliada

\begin{tabular}{|c|c|c|c|c|c|c|}
\hline & & & \multicolumn{4}{|c|}{ Exem plos } \\
\hline & & & Autom óvel & Ca dea do & Pe rfume & Museu \\
\hline$V$ & & $\begin{array}{l}\text { Valores } \\
\text { te rminais } \\
\text { (in tern o) }\end{array}$ & Amor & Proteção & Auto estima & Sabedoria \\
\hline$\hat{1}$ & & $\begin{array}{l}\text { Valores } \\
\text { ins trumentais } \\
\text { (externo) }\end{array}$ & União familiar & Segurança & Pert encim ento & Mente aberta \\
\hline $\mathrm{C}$ & $\begin{array}{l}\text { Nível de } \\
\text { abs tracão }\end{array}$ & $\begin{array}{l}\text { Conse qüuências } \\
\text { psicológicas }\end{array}$ & $\begin{array}{l}\text { Todos da família } \\
\text { se sentem bem }\end{array}$ & $\begin{array}{l}\text { Meus bens ficam } \\
\text { a salvo }\end{array}$ & $\begin{array}{l}\text { Impressiona } \\
\text { os outros }\end{array}$ & Mais cultura \\
\hline$\hat{4}$ & & $\begin{array}{l}\text { Conse qüiências } \\
\text { fun cion ais }\end{array}$ & Conforto & Resistente & $\begin{array}{l}\text { Imagem } \\
\text { sofisticada }\end{array}$ & $\begin{array}{l}\text { Conhecimento com } \\
\text { entret enimento }\end{array}$ \\
\hline$\downarrow$ & & $\begin{array}{l}\text { Atributos } \\
\text { abstratos }\end{array}$ & Mais espaço & Forte & Mais feminino & Interessante \\
\hline $\mathbf{A}$ & & $\begin{array}{l}\text { Atributos } \\
\text { con cretos }\end{array}$ & 5 portas & Pesado & $\begin{array}{l}\text { Formato da } \\
\text { embalagem }\end{array}$ & $\begin{array}{l}\text { Exposições bem } \\
\text { apresentadas }\end{array}$ \\
\hline
\end{tabular}

Fonte: representação proposta pelas autoras.

\section{Procedimentos da laddering}

Por permitir a identificação dos sistemas cognitivos dos indivíduos em níveis mais abstratos, a laddering foi escolhida para operacionalizar a teoria meios-fins. O psicólogo tenta chegar à raiz do problema, questionando; a laddering tem a mesma função com a exceção de que o profissional de marketing não está à procura da raiz do problema, mas, sim, tentando encontrar as razões para o consumidor comprar um determinado produto. Ao contrário de levantamentos, que avaliam ou delineiam sentimentos gerais do consumidor, a laddering avalia as razões mais profundas pelas quais o consumidor compra. "É uma forma de descascar uma cebola para superar o superficial e ir fundo no que é realmente importante" (Wansink, 2000, p. 30).

A técnica orienta a coleta e a análise de dados, e seus procedimentos são descritos neste artigo utilizando-se um exemplo hipotético, simplificado, sobre automóveis, que está baseado na Figura 1.

\section{Entrevista}

As questões são feitas, individualmente, ao entrevistado e tipicamente pede-se que ele reflita sobre as características capazes de diferir marcas e produtos entre si. Um número ótimo de entrevistas gira em torno de 30 (VRIENS; HOFSTEDE, 2000), embora haja pesquisas conduzidas via laddering com amostras menores, como a de Dibley e Baker (2001) e Foot e Lamb (2002). O pesquisador deve avaliar o grau de saturação e repetição das respostas ao decidir quantas entrevistas fazer.

Reynolds e Gutman (1988) explicam que as entrevistas podem iniciar-se de três modos:

a) a. "escolha de três" (triadic sorting): são apresentadas três marcas ao entrevistado, que indica as diferenças e semelhanças que duas delas apresentam em relação à terceira. Exemplo de questão: "Em que os carros A e B diferem do carro C?"

b) b. "diferenças de preferência de consumo": o entrevistado é solicitado a indicar as razões de sua preferência por uma marca específica. Exemplo de questão: "Qual é a marca de carro de sua preferência e por que ela é a preferida?" 
c) c. "diferenças de ocasião": procura-se contextualizar o consumo; pede-se ao entrevistado que mentalmente idealize o ambiente de compra do produto e descreva-o. Exemplo de questão: "Imagine-se na loja onde você comprou o carro A. Por que o carro A chamou a sua atenção?"

Todos esses estímulos buscam evidenciar as características mais importantes do produto. As preferências em relação às marcas e a descrição do consumo, em um dado contexto, são consideradas as evidências de atributos mais relevantes para o cliente. Com base nos atributos identificados, começa a ser construída a hierarquia de valor, o que implica as conseqüências serem reveladas a partir dos atributos, e os valores a partir das conseqüências.

Uma vez identificados os atributos, o pesquisador isola aqueles menos importantes e concentra a discussão nos mais importantes. Reynolds e Gutman (1988) comentam que, em média, cada respondente é capaz de mencionar de 10 a 12 atributos diferentes sobre uma dada categoria de produtos. $\mathrm{O}$ entrevistador, nesse caso, tem duas alternativas para selecionar os atributos mais importantes: (a) fazer a seleção dos atributos que parecem estar mais ligados à questão específica da pesquisa ou (b) pedir ao respondente que pontue as características segundo sua importância relativa, para, a partir de então, escolher os atributos com pontuações mais altas.

A partir desse momento, o entrevistado começa a ser conduzido à abstração, por meio de questionamentos sobre por que tal atributo (ou conseqüência) é importante. Sheth, Mittal e Newman (2001, p. 347) citam um exemplo para explicar como ocorre essa seqüência de perguntas, referindo-se a uma investigação sobre automóveis:

Por que essa característica é importante para você? Por exemplo, 'por que a aceleração rápida é importante para você?' Se a resposta for 'para manobras que me permitam fugir de engarrafamentos no trânsito', a pergunta seguinte é 'e por que isso é tão importante?', e assim por diante.

A entrevista laddering depende essencialmente das respostas dos entrevistados para seguir adiante, tendo como ponto de referência a pergunta "Por que é importante"?, até que níveis de abstração mais elevados sejam revelados.

\section{Análise dos dados}

A interpretação dos dados via laddering acarreta quatro fases: (a) análise de conteúdo, (b) desenvolvimento da matriz de implicação, (c) construção do mapa hierárquico de valor e (d) determinação das orientações de percepção dominantes.

a) Análise de conteúdo

As entrevistas são primeiramente transcritas e depois, cuidadosamente analisadas via exame do conteúdo. Nessa fase, as principais considerações expressas pelos respondentes são esclarecidas, e os dados são preparados para codificação, levando a um resumo de tudo que foi mencionado. Para isso, deve-se desmembrar os dados brutos e, em seguida, identificar e codificar os elementos.

O pesquisador deve alocar as partes do texto, com sentido semelhante, sob uma mesma oração. Por exemplo, se, em vários momentos do texto, o respondente manifestou que é importante prezar pelo bem-estar de sua família, essa idéia poderia ser identificada pela oração "todos da família se sentem bem". Pela leitura e análise do texto, o pesquisador é capaz de identificar as orações que estão interligadas e, assim, designar os elementos que compõem a hierarquia de valor. Uma vez especificados os atributos, que geralmente são as primeiras características mencionadas nas entrevistas, o pesquisador é capaz de reconhecer os demais elementos, guiando-se pela seqüência A-C-V. Exemplo: a característica "5 portas" está ligada à característica "espaço", que se conecta ao "conforto" e à oração "todos da família se sentem bem" - considerados conseqüências advindas dessas características. Um número é então atribuído a cada elemento, de acordo com a dimensão de valor à qual ele pertence. Assim, no início da contagem, o número 1 é didaticamente destinado a um atributo concreto. 
Ainda é importante salientar que o desmembramento de dados pode fornecer uma quantidade muito grande de orações e, portanto, de seqüências $\mathrm{A}-\mathrm{C}-\mathrm{V}$, o que poderia prejudicar a análise. O pesquisador deve ter sensibilidade para selecionar aquelas orações que são mais pertinentes à pesquisa. A análise de conteúdo é uma atividade interativa, onde os dados podem ser codificados e recodificados várias vezes, as categorias podem ser combinadas, divididas ou eliminadas, até que o analista se sinta seguro com o resultado (GENGLER; REYNOLDS, 1995). O Quadro 2 exemplifica o saldo dessa etapa:

Quadro 2. Resumo de códigos de conteúdo Exemplo representativo para automóveis

\begin{tabular}{llllll}
\hline Cód. & Atributos concretos & Cód. & Atributos abstratos & Cód. & Conseq. funcional \\
\hline 01 & Vidro elétrico & 05 & Mais espaço & 07 & Conforto \\
02 & Preço acessível & 06 & Versatilidade & 08 & Ter opções \\
03 & 5 portas & & & & \\
04 & Motor bi-combustível & & & & \\
\hline Cód. & Conseq. psicológicas & Cód. & Valores instrumentais & Cód. & Valores terminais \\
\hline 09 & Fazer economia & 12 & Responsabilidade para com a & 14 & Liberdade \\
10 & Todos na família se sentem & & família & 15 & Amor \\
& bem & 13 & União familiar & & \\
11 & Sentimento de & & & & \\
& independência & & & & \\
\hline
\end{tabular}

Fonte: representação proposta pelas autoras.

b) Matriz de implicação:

Uma tabela numérica, chamada matriz de implicação, é construída para representar as interações entre os elementos. Os códigos dos elementos são colocados nas linhas e nas colunas, e o interior da matriz é preenchido com números derivados da contagem de quantas vezes um dado elemento leva a outro. As relações entre os elementos podem ser diretas, quando são adjacentes, ou indiretas, quando há um outro elemento entre eles. Exemplo, o atributo "5 portas" (código 03) tem uma relação direta com o atributo "mais espaço" (código 05) e uma relação indireta com a conseqüência "conforto" (código 07) (ver Figura 1). A representação dessas relações é feita na matriz por meio de um ponto que separa as relações diretas à esquerda e as indiretas à direita. Assim, se as relações entre os elementos obedecem ao formato "xx.yy", as relações diretas seriam xx e as indiretas, yy. Pelo exemplo da Tabela 1, verifica-se que vinte e oito dos entrevistados mencionaram a conseqüência "todos da família se sentem bem". Tal número é obtido somando-se os valores das linhas e colunas referentes ao código 10. Seis entrevistados relacionaram diretamente os códigos 07 e 10 e apenas um respondente os relacionou indiretamente, daí o formato da célula 06.01 . 
Tabela 1. Matriz de implicação Exemplo representativo para automóveis

\begin{tabular}{|c|c|c|c|c|c|c|c|c|c|c|c|c|c|c|c|}
\hline Cód. & 01 & 02 & 03 & 04 & 05 & 06 & 07 & 08 & 09 & 10 & 11 & 12 & 13 & 14 & 15 \\
\hline 01 & & & & & & & 05.01 & & & 03.01 & & & 00.02 & & 8) \\
\hline 02 & & & & & & & & & 10.02 & & & 02.03 & & & \\
\hline 03 & & & & & 04.01 & & 01.04 & & & 01.06 & & & & & 7 \\
\hline 04 & & & & & & 06.03 & & 04.06 & 03.03 & & & & & & \\
\hline 05 & & & & & & & 03.00 & & & 01.04 & & & & 7 & \\
\hline 06 & & & & & & & & 03.00 & & 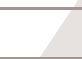 & 00.01 & & & 00.01 & \\
\hline 07 & & & & & & & & & & 06.01 & & & & & \\
\hline 08 & & & & & & & & & 05.00 & & 06.00 & & & 01.04 & \\
\hline 09 & & & & & & & & & & & & 04.01 & & & \\
\hline 10 & & & & & & & & & & & & & 05.00 & & \\
\hline 11 & & & & & & & & & & & & 7 & & 05.00 & \\
\hline 12 & & & & & & & & & & & & & & & 06.00 \\
\hline 13 & & & & & & & & & & & & & & & 04.00 \\
\hline 14 & & & & & & & & & & & & & & & \\
\hline 15 & & & & & & & & & & & & & & & \\
\hline
\end{tabular}

Fonte: representação proposta pelas autoras.

Leão e Mello (2001) questionam quantas vezes deve-se contar uma relação que foi mencionada mais de uma vez pelo mesmo entrevistado. A pergunta é: Se, em um momento da entrevista, o entrevistado relacionou 03 05 - 07, e, em outro momento, 03 - 05 - 10, quantas vezes a relação 03 - 05 deveria ser contada? Para não enviesar os resultados, Gengler (1995) explica que essa relação deveria ser considerada apenas uma vez.

A construção da matriz de implicação é um processo trabalhoso e complexo que gera uma série de dúvidas ao pesquisador. Pela minuciosidade dos inúmeros detalhes que envolvem a construção da matriz, o processo de elaboração da mesma é passível de erros. Para minimizar os riscos envolvidos em sua construção e manter a fidedignidade dos resultados, é recomendável o uso do LADDERMAP - software criado especificamente para auxiliar a operacionalização da análise da laddering.

O LADDERMAP poupa o pesquisador do trabalho manual envolvido na construção da matriz e reduz as chances de erros. Pelo fato de as interações entre os elementos serem calculadas, automaticamente e internamente, pelo software, faz-se desnecessária a apresentação da matriz de implicação quando o pesquisador utiliza o LADDERMAP. Se o software não for utilizado, a matriz de implicação deve ser tabulada a mão.

c) Construção do mapa hierárquico de valor (HVM):

O mapa hierárquico de valor é uma representação gráfica em forma de árvore que simboliza visualmente o agregado de respostas das fases anteriores. Com ele, tem-se uma visão geral do "raciocínio" dos clientes ao comprar ou escolher um produto. Nessa fase, Reynolds e Gutman (1988) e Valette-Florence e Rapacchi (1991) recomendam que a denominação ladder seja atribuída às seqüências eliciadas a partir dos respondentes, enquanto que o termo "cadeias" seja atribuído às seqüências de elementos que emergirem a partir da matriz de implicação.

Na construção do HVM, deve-se analisar a matriz de implicação, partindo-se da primeira linha e indo até a última. Para determinar quais serão as relações representadas no mapa, o analista deve estabelecer um ponto de corte. Por uma questão de estética, nem sempre é possível incluir todas as relações no desenho. Uma 
quantidade muito grande de relações pode tornar o mapa confuso e dificultar a análise. O ponto de corte é uma forma de priorizar as relações mais importantes. Cada célula da matriz deve ser comparada ao ponto de corte. Se a célula apresentar números iguais ou superiores a ele, a relação entre os elementos entrará no mapa, caso contrário, não.

No exemplo dos automóveis, considerando-se um ponto de corte igual a 3, verifica-se que a primeira coluna a apresentar relações superiores a 3 com a linha 01 é a coluna 07. Então, partindo-se até a linha 07, identifica-se que a coluna 10 é a que primeiro mostra relações superiores ou iguais ao ponto de corte, formando-se assim uma seqüência A-C-V. No exemplo, a seqüência formada é $01-07-10-13-15$. Esse processo deve-se manter até que a última linha seja atingida e todos os elementos tenham sido cobertos. A Figura 2 mostra o HVM. Entre parênteses estão os códigos dos elementos e, entre colchetes, o número de vezes que cada um foi mencionado pelos participantes.

Figura 2. Mapa hierárquico de valor

Exemplo representativo para automóveis

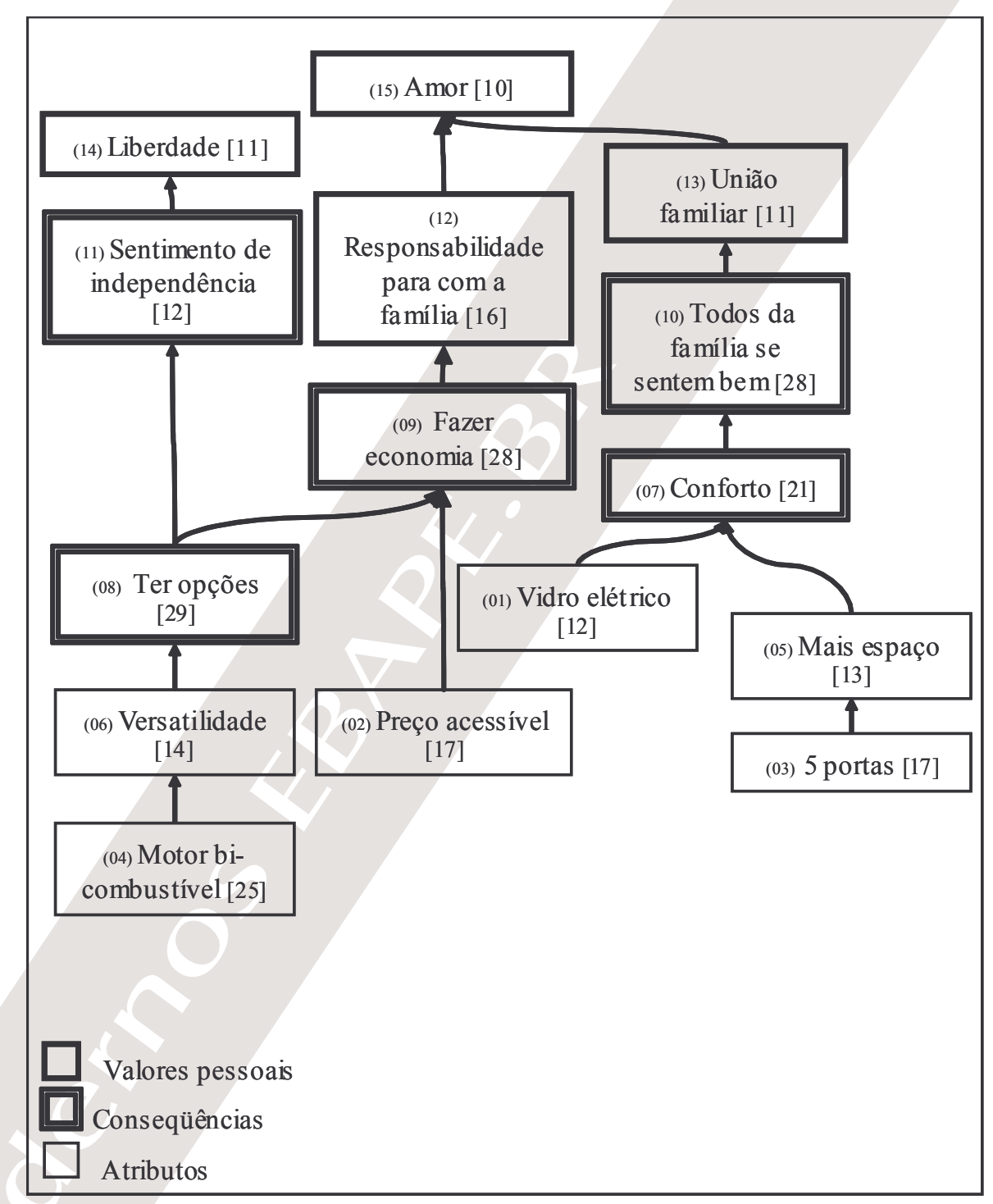

Fonte: representação proposta pelas autoras.

Nessa fase, é ainda preparada uma tabela que exprime todas as relações diretas e indiretas que partiram de um elemento e chegaram a ele. Oito relações diretas e quatro indiretas partiram do atributo "vidro elétrico" (código 01) e nenhuma veio até ele, como mostra a Tabela 2. Essa tabela é construída a partir da matriz de implicação, 
somando-se as linhas para identificar as relações que partiram do elemento e, as colunas para identificar as relações que vieram até ele.

Tabela 2. Resumo de relações diretas (xx) e indiretas (yy) Exemplo representativo para automóveis

\begin{tabular}{ccc}
\hline Código & De & Para \\
\hline 01 & 08.04 & 00.00 \\
\hline 02 & 12.05 & 00.00 \\
\hline 03 & 06.11 & 00.00 \\
\hline 04 & 13.12 & 00.00 \\
\hline 05 & 04.04 & 04.01 \\
\hline 06 & 03.02 & 06.03 \\
\hline 07 & 06.01 & 09.05 \\
\hline 08 & 11.12 & 07.06 \\
\hline
\end{tabular}

\begin{tabular}{ccc}
\hline Código & De & Para \\
\hline 09 & 04.01 & 18.05 \\
\hline 10 & 05.00 & 11.12 \\
\hline 11 & 05.00 & 06.01 \\
\hline 12 & 06.00 & 06.04 \\
\hline 13 & 00.00 & 05.02 \\
\hline 14 & 00.00 & 06.05 \\
\hline 15 & 00.00 & 10.00 \\
\hline & & \\
\hline
\end{tabular}

Fonte: representação proposta pelas autoras.

O HVM pode ser construído com o auxílio do LADDERMAP, que independe da contagem manual do número de relações diretas e indiretas e da identificação do tipo de relações. O software determina e calcula internamente todas essas interações, possibilitando uma série de alternativas para a definição do ponto de corte. O LADDERMAP, portanto, pode dispensar a elaboração da tabela que resume as relações diretas e indiretas dos elementos, pois desenha o mapa de acordo com o ponto de corte selecionado, conforme a decisão de mostrar as relações mais importantes, além de indicar automaticamente a quantidade de menções de um dado elemento.

d) Determinação das orientações de percepções dominantes:

As orientações de percepções dominantes dizem respeito às relações que mais contribuem para o resultado do mapa, e que, por isso, deveriam ser levadas em consideração em uma decisão de marketing. Uma forma de determiná-las é somar todas as relações diretas e indiretas que compõem as seqüências A-C-V, considerando-se as cadeias com maiores somas como as mais importantes. Entretanto, em concordância com Leão e Mello (2001), acredita-se que essa análise não deve priorizar o lado quantitativo. A importância das cadeias deve ser investigada, com cautela, pelo analista, levando-se em conta o contexto no qual a pesquisa foi feita e a categoria do produto.

\section{Restrições e recomendações}

A despeito das diversas vantagens da laddering, a técnica apresenta suas restrições:

\section{Na coleta de dados:}

- As perguntas repetitivas podem fazer com que as questões se tornem um tanto quanto óbvias ao respondente, além de tornar a investigação cansativa. Por essa razão, é importante informar o entrevistado das peculiaridades da técnica, deixando claro que os procedimentos adotados são parte de uma metodologia específica (WOODRUFF; GARDIAL, 2001).

- Na questão "por quê?", podem ocorrer níveis de abstração "artificiais", pois os entrevistados podem responder de maneira "racional", tentando encontrar argumentos para justificar seu comportamento (BOTSCHEN; THELEN; PIETERS, 1999).

- Existe possibilidade de o entrevistado não saber responder uma questão, por nunca haver pensado antes sobre ela ou por não conseguir refletir sobre as razões de sua importância (REYNOLDS; GUTMAN, 1988). 
- Usam-se questões demasiadamente pessoais que podem intimidar a expressão verdadeira do respondente, pois à medida que se vai subindo nos níveis da cadeia hierárquica de valor, as dimensões vão se tornando mais abstratas e mais pessoais (REYNOLDS; GUTMAN, 1988).

- Pode haver exigência de "entrevistadores com habilidades qualitativas" (VRIENS; HOFSTEDE, 2000, p. 6). Wansink (2000) adverte que algumas vezes as questões certas não aparecem facilmente e que o respondente pode se sentir nervoso ou desconfortável com a linha das questões, o que demanda habilidade do pesquisador ao conduzi-las.

- Há possíveis interferências do entrevistador na coleta de dados brutos e na análise de conteúdo (GRUNERT; GRUNERT, 1995). O processo subjetivo pode levar à eliminação de variáveis relevantes e, conseqüentemente, a estratégias de produto inapropriadas. O processo de simplificação das variáveis em categorias de atributos, conseqüências e valores pessoais também pode descartar insights interessantes (LIN, 2002).

\section{Gerais:}

- Há falta de critérios para o ponto de corte na construção do HVM (GRUNERT; GRUNERT, 1995, LIN, 2002).

- Ocorre impossibilidade de implementação em larga escala, por meio de amostras representativas, por serem entrevistas demoradas e de alto custo (VRIENS; HOFSTEDE, 2000).

- Há possibilidade de ocorrer interface pouco amigável do software LADDERMAP (LASTOVICKA, 1995).

- A construção da matriz de implicação é um processo trabalhoso e demorado (LASTOVICKA, 1995).

- A validade preditiva pode ser comprometida pelas limitações da coleta de dados e análise de conteúdo (GRUNERT; GRUNERT, 1995).

\section{Complementando o que foi encontrado na literatura, podem-se deduzir outras limitações:}

- O foco em aspectos positivos de atributos, conseqüências e valores, relegando os aspectos negativos para segundo plano. Atributos e conseqüências negativas podem afetar negativamente os valores pessoais. E em alguns produtos podem até ser mais fortes que os positivos.

- A aplicabilidade fora da área de marketing é restrita. Dos trabalhos pesquisados apenas os de Foote e Lamb (2002) e de Rugg et al. (2002) abordam temas não relacionados a marketing, enfocando, respectivamente, a ética e a cultura organizacional por meio da técnica.

Assim, percebe-se que o uso da laddering exige certos cuidados. Primeiro, deve-se verificar sua adequação ao problema. É desejável que o pesquisador tenha preparo e habilidade para realizar a entrevista e a análise dos resultados. Nesse ponto, ele deve discernir, de forma neutra, aquilo que realmente é relevante para a pesquisa, não se deixando levar pela simples aparência de uma primeira impressão que a análise de conteúdo pode suscitar. Por exemplo, o fato de alguns atributos, conseqüências e valores serem citados com maior freqüência não significa necessariamente que sejam os mais importantes, ou ainda, o fato de dois elementos não estarem diretamente relacionados não significa que não possam pertencer à mesma cadeia, pois pode haver entre eles uma relação indireta, ou eles podem se relacionar entre si por meio de ligações independentes, estabelecidas entre cada um deles e outros elementos.

\section{Considerações Finais}

A técnica laddering é extremamente útil e poderosa em marketing. Possibilita a descoberta de atributos, conseqüências e valores de forma mais estruturada e aprofundada que outras técnicas qualitativas disponíveis. A despeito disso, ainda é pouco utilizada, sendo seu uso, ao menos no Brasil, restrito aos círculos acadêmicos de pesquisa, quando também poderia ser melhor aproveitada em outros âmbitos. Considerando-se suas potencialidades, pode-se concluir que a carência de estudos pode estar ligada ao desconhecimento e à falta de maior disseminação da técnica. 
Há inúmeras possibilidades de pesquisa em marketing explorando mais a técnica. Por exemplo, seria possível promover discussões em grupos em vez de entrevistas individuais para identificação de diversas cadeias, considerando tanto atributos e conseqüências positivas quanto negativas. Isso poderia ser feito antes do lançamento de um produto, para analisar sua viabilidade. A incorporação de ferramentas de análise, como softwares e outras técnicas qualitativas e quantitativas, é um aspecto que também deveria ser mais explorado. Além do uso acadêmico, a laddering também poderia ser mais utilizada em empresas para estudos de estratégias de marketing como desenvolvimento de produtos, comunicação, segmentação, posicionamento e planejamento. Apenas para ilustrar essas possibilidades, seguem alguns exemplos.

a) Desenvolvimento de produtos: Podem-se projetar produtos enfatizando os atributos que se sobressaírem na avaliação. Além disso, novos produtos podem ser idealizados, tendo em vista as conseqüências e valores que os consumidores estão buscando.

b) Comunicação: As mensagens podem enfatizar os atributos de bens e serviços mais valorizados pelos consumidores e também reforçar as associações estabelecidas entre as dimensões de valor (REYNOLDS; WHITLARK, 1995).

c) Segmentação: A laddering também é útil na segmentação de mercado. Por estruturar as dimensões de valor sob o mesmo formato A-C-V, as comparações entre grupos são facilitadas, sendo possível traçar paralelos entre diferentes culturas (DIBLEY; BAKER, 2001) ou perfis de consumidores, como atuais e potenciais (GUTMAN, 1982). Da mesma forma é viável agrupar clientes com cadeias ou valores pessoais semelhantes.

d) Posicionamento: O posicionamento do produto ou da marca pode, por sua vez, ser planejado com base em quaisquer das três dimensões de valor e com base em suas importâncias relativas para o cliente.

e) Planejamento de marketing: Ao conhecer em profundidade o comportamento dos consumidores, o profissional de marketing tem condições de planejar melhor suas estratégias, oferecendo produtos e serviços mais adequados a cada perfil de cliente.

Este artigo procurou explicar como a laddering está inserida no contexto da pesquisa de marketing (tópicos 1, 2 e 3), descrever e exemplificar os procedimentos da técnica (tópico 4), relatar as impressões de pesquisadores que já a utilizaram, bem como as próprias experiências das autoras (tópico 5) e, principalmente, contribuir para sua disseminação, indicando usos e realçando sua utilidade em marketing (tópico 6). As aplicações da laddering em marketing são ricas e variadas. É salutar para a área que pesquisadores e profissionais de mercado estejam abertos ao uso de técnicas qualitativas que, além de proporcionar conhecimentos e informações valiosas sobre o consumidor, também apóiam decisões. 


\section{Referências bibliográficas}

ALLEN, M. W. A practical method for uncovering the direct and indirect relationships between human values and consumer purchases. The Journal of Consumer Marketing, Santa Barbara, v. 18, n. 2, p. 102-113, 2001.

ASSAEL, H. Consumer behavior and marketing action. Cincinnati: South-Western College Publising, 1995.

BAGOZZI, R. P.; DABHOLKAR, P. A. Consumer recycling goals and their effect on decisions to recycle: a means-end chain analysis. Psychology \& Marketing (1986-1998), v. 11, n. 4, p. 313-341, July/Aug. 1994.

Discursive psychology: an alternative conceptual foundation to means-end chain theory. Psychology \&t Marketing, Hoboken, v. 17, n. 7, p. 535-556, July 2000.

BANNISTER, D.; MAIR, J. M. The evaluation of personal constructs. London: Academic Press. 1968.

BOTSCHEN, G.; THELEN, E. M.; PIETERS, R. Using means-end structures for benefit segmentation an application to services. European Journal of Marketing, Bradford, v. 33, n. 1-2, p. 38-58, 1999.

CHRISTENSEN, G. L.; OLSON, J. C. Mapping consumer's mental models with ZMET. Psychology \& Marketing, New York, v. 19, n.6, p. 477489, June 2002.

COLLEN, H.; HOEKSTRA, J. Values as determinants of preferences for housing attributes. Journal of Housing and the Built Environment, Delft, v. 14, n. 3-4, p. 285-306, Sep. 2001.

DIBLEY, A.; BAKER, S. Uncovering the links between brand choice and personal values among young British and Spanish girls. Journal of Consumer Behaviour, London, v. 1, n. 1, p. 77-93, June 2001.

FOOTE, D.; LAMB, K. Eliciting information about the values of HRM practitioners using laddering interviews. Business Ethics: a European Review, Oxford, v. 11, n. 3, p. 244-252, 2002.

GENGLER, C. E.; HOWARD, D. J.; ZOLNER, K. A personal construct analysis of adaptive selling and sales experience. Psychology \& Marketing (1986-1998), v. 12, n. 4, p. 287-304, July 1995.

GENGLER, C. E.; MULVEY, M. S.; OGLETHORPE, J. E. A means-end analysis of mother's infant feeding choices. Journal of Public Policy \& Marketing, Ann Arbor, v. 18, n. 2, p. 172-188, Fall 1999.

GENGLER, C. LADDERMAP user's manual. Camden, New Jersey, 1995.

GENGLER, C.; REYNOLDS, T. Consumer understanding and advertising strategy: analysis and strategic translation of laddering data. Journal of Advertising Research, New York, v. 35, n. 4, p. 19-32, July/Aug. 1995.

GOLDENBERG, M. A.; KLENOSKY, D. B.; O'LEARY, J. T.; TEMPLIN, T. J. A means-end investigation of ropes course experiences. Journal of Leisure Research, v. 32, n. 2, p. 208-224, 2000.

GRUNERT, K. G.; GRUNERT, S. C. Measuring subjective meaning structures by the laddering method: theoretical considerations and methodological problems. International Journal of Research in Marketing, Amsterdam, v. 12, n. 3, p. 209-225, 0ct. 1995.

GUTMAN, J. A means-end chain model based on consumer categorization processes. Journal of Marketing, Chicago, v. 46, p. 60-72, Apr. 1982.

HINKLE, D. N. The change in personal constructs from the viewpoint of a theory of implications. Ph.D. Thesis (não publicado), Ohio State University, 1965.

IGNÁCIO, C. P. O comportamento do consumidor de produtos alimentícios: um estudo exploratório sobre a importância das marcas. Dissertação (Mestrado em Administração) - Departamento de Administração da Pontifícia Universidade Católica de São Paulo. São Paulo: PUC, 2003.

KELLY, G. A. The psychology of personal constructs (v. 1). New York: Norton, 1955.

KENNEDY, K. N.; GOEBEL, D. J.; DEETER-SCHMELZ, D. R. Value laddering as a method of sales research. AMERICAN MARKETING ASSOCIATION CONFERENCE, 11, 2000, Proceedings... AMA, 2000.

LANGERAK, Fred; PEELEN, Ed; NIJSSEN, Ed. A laddering approach to reduce the cycle time of new-to-the-firm products. Journal of Product Innovation Management, v. 16, n. 2, p. 173-182, March 1999.

LASTOVICKA, J. L. LADDERMAP: version 4.0 by Chuck Gengler. Journal of Marketing Research, Chicago, v. 32, n. 4, p. 494-497, Nov. 1995. 
LEÃO, A. L. M. de S.; MELLO, S. C. B. Mensurando customer value através do método laddering: uma proposta de aplicação da técnica para o ambiente virtual. In: ENCONTRO NACIONAL DA ASSOCIAÇÃO NACIONAL DOS PROGRAMAS DE PÓS-GRADUAÇÃO EM ADMINISTRAÇÃO, 25, 2001, Anais... Campinas, ANPAD, 2001.

; C. Conhecendo o valor do cliente virtual: uma análise utilizando a teoria de cadeias de meios-fins. ENCONTRO NACIONAL DA ASSOCIAÇÃO NACIONAL DOS PROGRAMAS DE PÓS-GRADUAÇÃO EM ADMINISTRAÇÃO, 26, 2002, Anais... Salvador, ANPAD, 2002.

LIN, C. F. Attribute-consequence-value linkages: a new technique for understanding customer's product knowledge. Journal of Targeting, Measurement and Analysis for Marketing, London, v. 10, n. 4, p. 339-352, June 2002.

MANYIWA, S.; CRAWFORD, I. Determining linkages between consumer choice in a social context and the consumer's value: a means-end approach. Journal of Consumer Behavior, v. 2, n. 1, p. 54-70, 2002.

OLSON, J.; REYNOLDS, T. Understanding consumer's cognitive structures: implications for advertising strategy. In: PERCY, L.; WOODSIDE, A. Advertising and Consumer Psychology. MA: Lexington, 1983.

ORSINGHER, C.; MARZOCCHI, G. L. Hierarchical representation of satisfactory consumer service experience. International Journal of Service Industry Management, v. 14, n. 2, p. 200-216, 2003.

REYNOLDS, T. J.; GUTMAN, J. Laddering theory, method, analysis, and interpretation. Journal of Advertising Research, New York, v. 28, p. 11-31, Feb./Mar. 1988.

REYNOLDS, T.; WHITLARK, David. Applying laddering data to communications strategy and advertising practice. Journal of Advertising Research, New York, v. 35, n. 4, p. 9-16, July/ Aug. 1995.

ROKEACH, M. The nature of human values. New York: Free Press, 1973.

RUGG, G.; EVA, M.; MAHMOOD, A.; REHMAN, N.; ANDREWS, S.; DAVIES, S. Eliciting information about organizational culture via laddering. Information Systems Journal, v. 12, p. 215-229, 2002.

SHETH, J. N.; MITTAL, B.; NEWMAN, B. Comportamento do cliente: indo além do comportamento do consumidor. São Paulo: Atlas, 2001.

THYNE, M. The importance of values research for nonprofit organisations: the motivation-based values of museum visitors. International Journal of Nonprofit and Voluntary Sector Marketing, v. 6, n. 2, p. 116-30, 2001.

VALETTE-FLORENCE, P.; RAPACCHI, B. Improvements in means-end chain analysis: using graph theory and correpondence analysis. Journal of Advertising Research, New York, p. 30-45, Feb./March. 1991.

VELUDO-DE-OLIVEIRA, T. M. Valor em serviços educacionais: um estudo sobre o marketing educional no contexto da teoria de cadeias meios-fim. Dissertação (Mestrado em Administração) - Departamento de Administração da Faculdade de Economia, Administração e Contabilidade da Universidade de São Paulo. São Paulo, USP, 2003.

VRIENS, M.; HOFSTEDE, F. T. Linking attributes, benefits and consumer values. Journal of Marketing Research, Chicago, v. 12, n. 3, p. 4-10, Fall 2000.

WANSINK, B. New techniques to generate key marketing insights. Journal of Marketing Research, Chicago, v. 12, n. 2, p. 28-36, Summer 2000.

Using laddering to understand and leverage a brand's equity. Qualitative Market Research: An International Journal, v. 6, n. 2, p. 111-118, 2003.

WOODRUFF, R. B.; GARDIAL, Sarah F. Know your customer: new approaches to understanding customer value and satisfaction. Malden: Blackwell, 1996. 